\title{
A Comparative Study of "Taihu Stone" Image in Painting and Ceramic Decoration
}

\author{
Lejun Liu ${ }^{1, *}$ Qi Xu ${ }^{1}$ \\ ${ }^{1}$ Jingdezhen Ceramic Institute, Jingdezhen, Jiangxi 333403, China \\ *Corresponding author. Email: llj7822000@126.com
}

\begin{abstract}
"Taihu Stone" is an important image element in Chinese traditional culture. It plays not only a special role in traditional Chinese painting, but also in ceramic decoration. This article compares the artistic characteristics of the image in each period and the aesthetic connotation behind it, and uses the comparison of traditional painting and ceramic decoration "Taihu Stone" image as the context to reveal the inheritance and transformation of the "Taihu Stone" image. And it further explore the "Taihu Stone" image changes caused by the evolution of social forms and spiritual thinking, in order to find reference and inspiration for ceramic decoration art design.
\end{abstract}

Keywords: "Taihu Stone" image, Chinese painting, Ceramic decoration.

\section{INTRODUCTION}

"Taihu Stone" is named because it is abundant in the Taihu Lake area. Its exquisite and translucent shape and the appearance of heavy mountains are one of the typical traditional stone offerings, reflecting the aesthetic characteristics of "wrinkle, leakage, thinness, and transparency". The Taihu Stone Collection is naturally crafted and made to win with its shape. Stone appreciation has also become an elegant entertainment method for emperors, generals, and scholars. At the beginning of the art of Western stone, stone carvings from the ancient Greek and Roman period mostly showed the appeal of Western religious theology and advocacy and mystery. The formation of the image of "Taihu Stone" in Chinese classical art occurred from people's worship and love of natural mountains and rivers. The development of the "Taihu Stone" image is inseparable from the specific relationship between Chinese classical aesthetics, social life and cultural levels.

The theory of Chinese stone appreciation art originated from the pre-Qin thinker Zhuangzi's definition of the category of "beauty and ugliness". Zhuangzi believed that the "beauty" and "ugliness" in artistic images were not opposed, but were unified and transformed into each other in the whole picture, and even thought that the "ugly" image could highlight its vitality. This kind of inner aesthetic state has opened up people's aesthetic vision and aesthetic orientation. If the cultural connotation of the "Taihu Stone" image is ignored, its image is not round and irregular. But under the influence of such aesthetic thinking, the image of "Taihu Stone" has become more emotional and more profound.

\section{IMAGE FEATURES OF "TAIHU STONE" IN CHINESE PAINTINGS}

The study of the "Taihu Stone" image in Chinese painting art cannot be separated from the human beauty of Chinese classical art. The "Taihu Stone" image not only inherits the "real" of natural beauty, but also reveals the "emptiness" full of "qi". The combination of virtuality and reality advocated by Chinese classical aesthetics is fully manifested in a piece of stone, giving people the joy of soul and silent company. Such a "human nature" stone is transformed into a "Taihu stone" image into the artwork, which not only reflects the characteristics of the image, but also incorporates different definitions and expressions. 


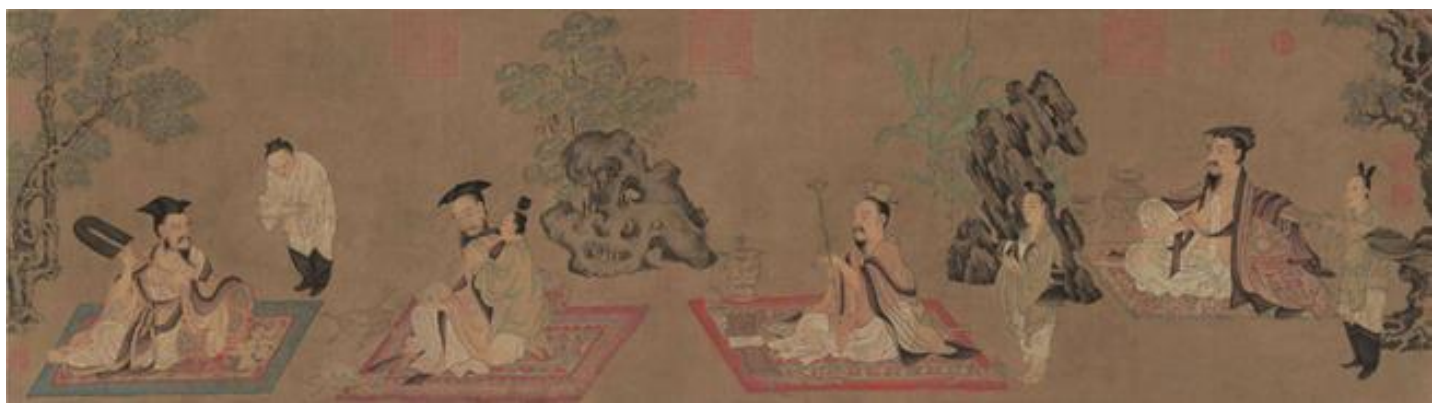

Figure 1 Gao Yi Tu(detail), Ink and color on silk, painted by Sunwei, in Tang Dynasty, collected in Shanghai Museum.

The paintings of mountain and rock appeared in the Wei, Jin, Southern and Northern Dynasties. The paintings of mountains and rocks in Luo Shen $\mathrm{Fu}$ Tu by the great painter of the Eastern Jin Dynasty Gu Kaizhi painted a series of mountain and rock images. Background and vassal, this form of blending nature with the scene of the characters can show the thinking tendency of pursuing the status of natural beauty in the Wei and Jin Dynasties. Later in the Tang Dynasty Sun Wei's "Gao Yi Tu" ("Figure 1"), the image of "Taihu Stone" appeared in the theme of "Seven Sages in the Bamboo Forest", which also showed artistic charm of the
Wei and Jin Period. The image of "Taihu Stone" and the trees in the painting are still supporting roles, but the image of "Taihu Stone" in the painting is vivid and exquisite in shape. The author tends to the composition format of "one person, one stone" when arranging the picture, which reflects the contrast of this period. Appreciation and attention to natural beauty, but as far as the overall image of the picture is concerned, the "Taihu Stone" image and the picture have a low degree of integration. They still exist as accessories for the characters and do not reflect high aesthetic consciousness.

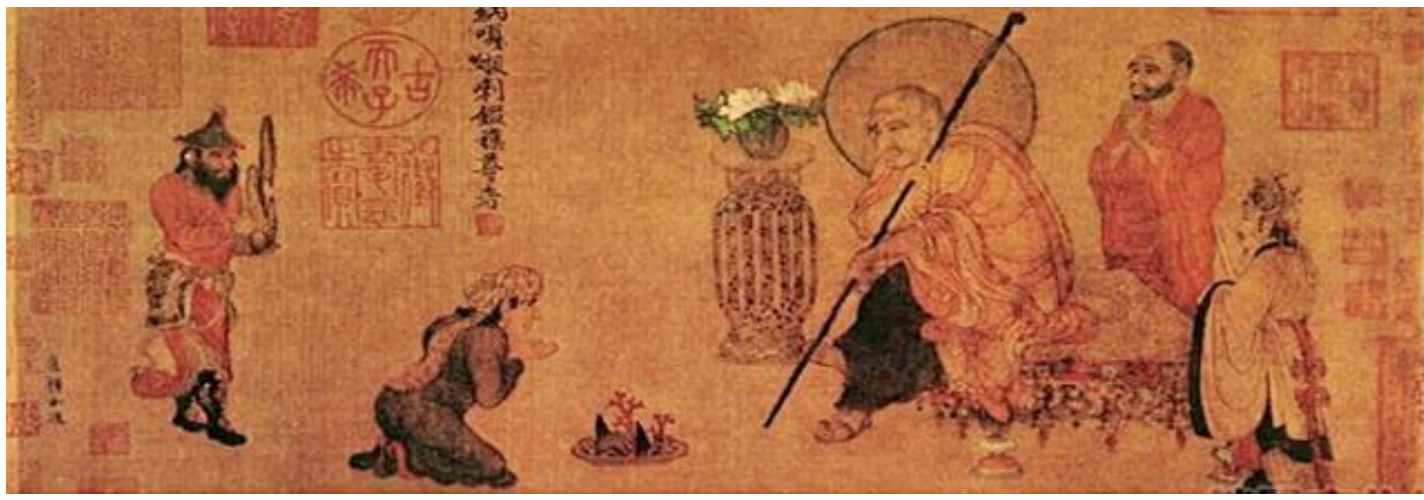

Figure 2 Picture of the Six Venerables (Song Dynasty copy) Ink and color on silk, painted by Lu Luojia, in Tang Dynasty, collected in the Palace Museum, Beijing.

Also in the Tang Dynasty, the image of "Taihu Stone" in the painting was transformed from the natural landscape background to the stone appreciation and bonsai in the figure painting. In the "Six Venerables" ("Figure 2"), the "Taihu Stone" image is from the reclusive master The back of is transferred to the hands of the worshippers. On the left side of the screen, the two worshippers are standing and holding a tall and tall Taihu stone, and the other is kneeling. A pot of bonsai composed of strange stones and corals is placed on the ground in front of them. The look is pious. As far as the "Taihu Stone" image is concerned, the performance in the painting "Six Masters" is more harmonious, and the combination with the main body of the picture and the plot of the image is more natural. The image of "Taihu Stone" has also changed from a pure natural background to an important role that affects the overall weather of the picture.

Ordinary people's perception of stone is "dry", it has no vital signs and no fresh form. However, under the influence of classical philosophy and aesthetics, it is precisely because of its "dryness" that it shows its "immortality" characteristic. This is consistent with the idea of "Neither birth nor death" in Lao Zhuang's philosophy and Mahayana 
Buddhism. The image of "Taihu Stone" is dry and full of vitality, just like the ganoderma of the earth, it is a symbol of life. The image of "Taihu Stone" in the painting "Six Venerables" is an interpretation of this concept.

\section{THE IMAGE OF "TAIHU STONE" IN CERAMIC DECORATION WORKS}

Ceramic decoration began with the historical development of porcelain. The ceramic decoration method with painting as the mainstream was seen in the Tang and Song dynasties, and flourished in the Yuan Dynasty and beyond. The ceramic decoration materials come from different genres such as traditional Chinese paintings and prints of the same period. The "Taihu Stone" image has been processed by ceramic decoration materials, and it has a very different appearance from other materials. Through comparative analysis of ceramic painting works, we can see the composition of the "Taihu Stone" image in different shapes and the different decorative effects of different craftsmanship paintings.

\subsection{Performance of Composition}

The influence of Chinese painting on ceramics is relatively obvious. The blue and white dragon and phoenix pattern cover box in the Ming and Wanli period, the main body is the dragon and phoenix moire pattern at the center of the cover, and the "Taihu stone" image is combined with pine, bamboo, plum and ganoderma on the waist of the cover. Plants are combined into a decorative belt, inheriting the tradition of using "Taihu stone" as a supporting role in early Chinese paintings. In the later ceramic compositions, this decorative format is still retained, coexisting with other decorative composition forms using the image of "Taihu Stone".

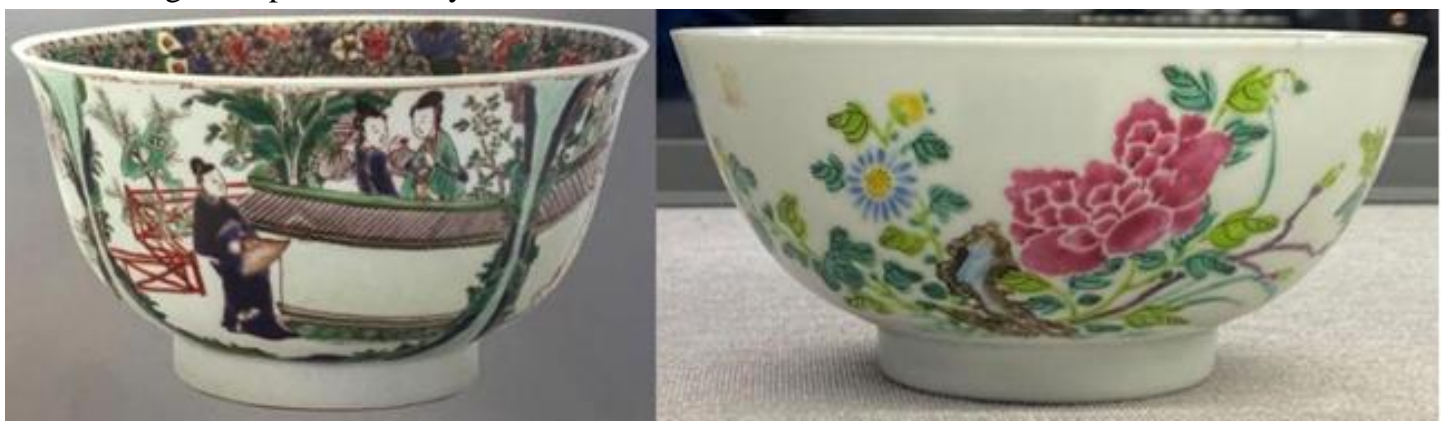

Figure 3 A bowl with colorful figures, Kangxi period of the Qing Dynasty, Collected in Jieruitang (Left) and Pastel peony flower pattern bowl, Yongzheng period of the Qing Dynasty (Right).

The image of "Taihu Stone" in Kangxi's colorful Western Chamber figure bowl (the left part of "Figure. 3") is used as a decorative supporting role, with light colors and small areas dotted on the edge of the opening. This kind of picture composition conforms to the usual layout of character story pictures, which shows the inheritance of the theme of character story pictures in ceramic paintings. In the Yongzheng period of the Qing Dynasty, which advocated elegant aesthetics, the image of "Taihu Stone" was also decorated with popular pastels (the right part of "Figure 3"), but in the composition form, the image of "Taihu Stone" was decorated with plant images such as peony Overall, it fully highlights the importance of the image in the subject of flower and bird painting. In the composition of the longevity plate with flower and fruit pattern in the Yongzheng period of the Qing Dynasty, the image of "Taihu Stone" is placed directly in front, which shows the high status of the image. Putting the heavy natural material image of the "Taihu Stone" image directly below it plays the role of lining the center of the picture, providing a center of gravity and a sense of balance for the circular composition. It can be seen that the composition of the image in the ceramic works is not the same shape, but changes according to the overall picture requirements.

\subsection{Performance of Modeling}

The image of "Taihu Stone" was originally an imitation and evolution of natural material. In the traditional Chinese paintings, the "Taihu Stone" image was transformed into a strangely shaped table stone in the "Nansheng Lu Si Le Tu" and "Character Picture" by Chen Hongshou in the Ming Dynasty. Although the chair also appeared with literati scholars, the relationship between "stone and 
man" is more harmonious. It reflects the deepening of the aesthetic view of the "Taihu Stone" image. And this deepening is also reflected in the ceramic works. In the Kangxi Alum Red-painted Gold Eight Immortals and Longevity Stars Plate ("Figure 4") in the Qing Kangxi, the "Taihu Stone" image is turned into a quaint stone bench for the incense burner, which the connection between the image of "Taihu Stone" and the characters draws closer. From the stone bench and the stone bench, we can see the transformation of the practicality of the "Taihu Stone" image in the ceramic works, and we can see the different tendencies of decorative demands in the ceramic works.

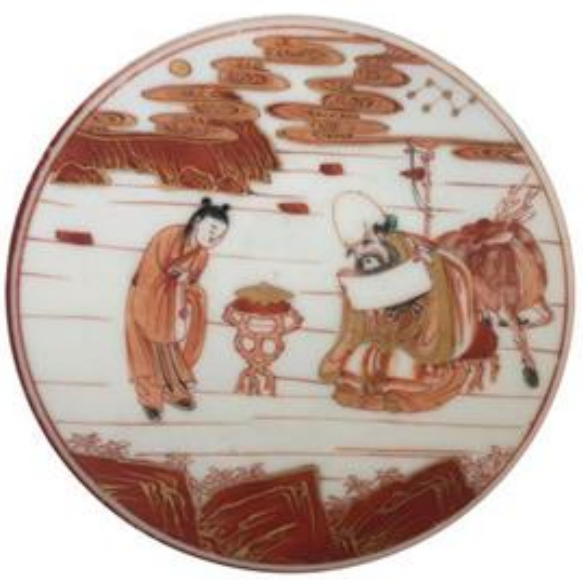

Figure 4 A plate with colorful figures painted in gold on alum during the Kangxi period of the Qing Dynasty (partial), collected in Jieruitang, the United States.

\subsection{Performance of Craftsmanship and Decoration}

It can be seen from the ceramic works that the image of "Taihu Stone" has its own characteristics due to the different decorative craftsmanship. Take the craftsmanship of colorful, blue and white, bucket color and pastel color as examples. The "Taihu stone" image of the Kangxi multicolored porcelain mostly used the single-line flat coating method. The "Taihu stone" image in the figure bowl of the colorful Western Chamber of the Kangxi Emperor of the Qing Dynasty was not in the main decoration position, so only the ancient water green was used for flat coating. The picture on the plate of Kangxi Alum Red Drawing with Gold and Eight Immortals and Longevity Stars in the Qing Dynasty is mainly painted in alum red, the large stone directly below is painted with gold lines to increase the sense of chaos, and the stone bench in the center of the picture is lightly stained with pale alum red ("Figure 4"), To open up the hierarchical relationship between.

The basic shape of the "Taihu stone" image in the blue and white porcelain is not much different. The works are the same for birthday celebrations. The longevity dial with a flower and fruit pattern in the Yongzheng period of the Qing Dynasty is outlined in a single line, and the relationship between light and dark is drawn using the blue and white water separation technique, which is a decorative effect. It is relatively thin; the shape and color of the "Taihu stone" image in these three crafts are not the Taihu stone in the natural image, but the technological characteristics presented after the adaptation of ceramic materials, with the image of the ceramic work "Taihu stone" Features. From the perspective of pastel decoration craftsmanship, the "Taihu stone" image of the Yongzheng famille rose peony flower pattern bowl in the Qing Dynasty is closer to its natural form. The pearl material is used as the base and two layers of pastel material are used to cover it to give it a sense of volume. The vivid color is inseparable from the material properties of pastels.

From the comparison of ceramic works, it is not difficult to see that the image of "Taihu Stone" is widely used in ceramic decoration. The subject matter covers character stories, flower and bird pictures, birthday wishes, etc. In the form of decoration, it is not only used as a decorative belt on the shoulder and waist of the utensil, but it also appears as the main body of the screen. Especially in the Ming and Qing Dynasties when ceramic decoration was mature, people had a deeper understanding of the aesthetic culture contained in the image of "Taihu Stone". 


\section{THE SIMILARITIES AND DIFFERENCES OF "TAIHU STONE" IMAGES IN PAINTING AND CERAMIC DECORATION}

A comparative study of the "Taihu Stone" images in traditional painting and ceramic decoration shows that there are many differences between the two due to the difference in materials and craftsmanship. In traditional paintings, the decorative techniques of stone are often represented by the method of chasing, such as axe chopping and draping. The color is based on the natural form of Taihu stone, which is dark brown or yellowish brown; while the image of "Taihu stone" in the ceramic decoration is completely different. The morphological characteristics of "Taihu stone" image are refined and generalized. The colors used are painted according to different ceramic decoration techniques, such as blue and white blue, alum red, and Kangxi multicolored green. The image of "Taihu Stone" under this decoration is slightly exaggerated, but it has an elegant and hazy natural beauty.

The painting of the "Taihu Stone" image and the shape and color of the ceramic decoration are unified in the pastel craftsmanship. This is due to the particularity of the pastel materials, and the inheritance between the two can also be seen. In addition to natural forms in traditional painting, there are also practical changes such as stone tables and chairs, but such changes are produced after a long period of practice and ideological transformation. In ceramic decorations with a relatively short development time, you can see the coexistence of multiple "Taihu stone" image forms in the same period. The scenes and the "role" they play all prove that the "elegance" in the aesthetics of the image has been transferred to ceramics. In terms of decoration, ancient porcelain painters do not necessarily understand the light, tenacious vitality and aesthetic origins of the "Taihu Stone" images, but they can be used in ceramic decoration works. It can be seen that the "Taihu Stone" images have profound influence in multi-dimensional art forms.

\section{CONCLUSION}

The comparative study of the "Taihu Stone" image is based on the cultural origin of the image and its evolution from traditional painting to ceramic painting form. Comparing the image expression of "Taihu Stone" in traditional painting and ceramic decoration, it can be seen that in traditional painting, the classical aesthetic consciousness has influenced the literati painting and realized the evolution from the supporting role to the protagonist. This transition is long. From the perspective of the timeline, ceramic decoration was the most outstanding decoration material in the Ming and Qing Dynasties. Its "Taihu stone" image decoration performance absorbed the experience of traditional painting, and flexibly applied various forms of expression in different decoration scenes. As ceramics is a practical material, the high frequency of the decoration of the "Taihu Stone" image also confirms the universality of the image, and proves that the aesthetic connotation and artistic tension in the "Taihu Stone" image are worthy of appreciation. And provide artistic reference and theoretical basis for future design.

\section{AUTHORS' CONTRIBUTIONS}

This article is completed by Lejun Liu and Qi $\mathrm{Xu}$. Lejun Liu is responsible for revising and editing, and Qi Xu wrote the manuscript.

\section{REFERENCES}

[1] Zong Bing. Wang Wei, "Preface to Painting Landscapes" $[\mathrm{M}]$ People's Fine Arts Publishing House, 1985

[2] Liu Jinghong. Stone Painting and Appreciation [M] Wuhan University Press, 2006

[3] Zhu Liangzhi. The life spirit of Chinese art [M] Anhui Education Publishing House, 2006

[4] Ding Wenfu. Ancient Chinese Stone Appreciation [M] SDX Joint Publishing Company, 2002

[5] Zhang Sheng. Taihu Stone Aesthetic Art in Chinese Painting [J] Garden Theory and Research, 2014

[6] Zhu Liangzhi. The Merry of Stone [M] ZHONGHUA Book Company, 2016

[7] Zhu Guangqian. Tan Mei [M] Chinese Writer Publishing House, 2018 